\title{
Negoziare sulla migrazione digitale. Conversazione con Roberto Casati
}

\author{
di Enrico Manera
}

II recente Contro il colonialismo digitale di Roberto Casati ha aperto un vasto dibattito sul cambiamento epocale comportato dalla massiccia diffusione del digitale, anche in relazione alla storia culturale della lettoscrittura e alla sua correlazione con l'educazione e I'insegnamento. Scartando di lato rispetto alla contrapposizione tra apocalittici e integrati, tra il "rifiuto luddistico» dei tecnoclasti e l' "adorazione messianica» dei tecnofili, il libro muove dal presupposto che si tratta di negoziare con l'innovazione digitale e lavorare seriamente sull'educazione a fronte di un paesaggio umano, cognitivo e sociale rapidamente mutevole.

Contro il colonialismo digitale ha avuto una vasta risonanza e ha il merito di aver sollevato una autorevole voce di critica costruttiva e non passatista alla questione della trasmigrazione dal cartaceo al digitale.

Prima di tutto tengo a puntualizzare che non è mia intenzione fare della denuncia, vorrei evitare di diventare una bandiera della resistenza contro la scuola digitale o l'e-book. Dall'altro lato contro la retorica martellante della stampa e delle istituzioni, che spesso paiono dei comunicati stampa dei produttori di hardware e software, mi sembra più utile fornire strumenti soprattutto agli insegnanti e ai genitori. Proporre un lavoro costruttivo. Che parta da spunti empirici e spunti concettuali, nel senso della chiarificazione e di ridefinizione e di uso delle parole giuste. Ad esempio, non ci sono dei 'nativi digitali', al limite dei 'nati digitali' (o dei coatti digitali); non esiste il 'multitasking' ma uno zapping che si riduce ad essere 'taskswitching'. Bisogna evitare l'idea che l'assuefazione ai nuovi media digitali sia scontata e produca automaticamente competenze ; bisogna prendere con estrema cautela l'idea di un nuovo modello antropologico inevitabile e ineluttabile. Le nuove generazioni esposte al digitale non sono né malati né mutanti. Dal punto di vista cognitivo sono esattamente come erano i nostri antenati del paleolitico, ma sono in un ambiente diverso, con un diverso design.

\section{Partiamo ad esempio dalla questione libro cartaceo/e-book: ha senso pensare in termini di antagonismo ed esclusione?}

C'è libro e libro naturalmente. II libro che abbiamo conosciuto per secoli, quello stampato e rilegato, è in realtà tanti tipi di oggetti diversi. Per esempio il libro in cartaceo non ha più ragion d'essere se pensiamo a cosa è una enciclopedia. Quel formato fatto di pagine chiuse tra due copertine, per contenere una informazione puntuale, oggi, richiederebbe centinaia di chilometri di scaffali. Questo è un tipo di contenuto la cui migrazione fuori dal formato copertina/rilegatura risulta vantaggiosa. Ma pensiamo prima di tutto a cos'è un libro. Di solito il libro viene considerato come una tecnologia antica di stoccaggio di informazione, come una tecnica per congelare, conservare e esternare memoria. lo contesto questa riduzione del libro a tecnologia di stoccaggio di memoria, è anche questo naturalmente, ma è soprattutto una tecnologia del riesame. Qualcosa che permette di presentare informazione e argomenti in modo tale che chi li presenta sa che il 
lettore li potrà riesaminare con più facilità, e anche indicizzare e analizzare con un maggior effetto di mantenimento dell'attenzione. Ed è esattamente questo il pregio del libro cartaceo, nel momento in cui la nostra attenzione va difesa da brusìo e da eccesso di informazione, come i pozzi artesiani dall'inquinamento. Il libro di carta è qualcosa che ti dice implicitamente: - Se tu non mi lasci io non ti distraggo -. E questo condiziona la scrittura, la qualità della scrittura pensata per l'argomentazione. Quindi mantenere in vita libri di carta significa mantenere in vita scrittori di libri di carta, creare quella particolare modalità di interazione tra scrittore/lettore caratteristica della saggistica. Non a caso la competizione con stimoli di altra natura sta già cambiando tutto questo: gli editori chiedono meno testo, su libri e giornali la richiesta è quella di fare piccoli riquadri detti 'di approfondimento' ma in realtà di distrazione. Questa pillolizzazione del libro, la tendenze della riduzione delle righe e verso i piccoli moduli significa anche perdere in qualità della scrittura e dunque dell'organizzazione della conoscenza. Un libro ha dunque queste caratteristiche: modalità intensiva, stoccaggio dell'informazione, tecnologia del riesame, qualità della scrittura, contratto sull'attenzione.

Al di là della economia dell'attenzione, costantemente minacciata in un ecosistema come il tablet sempre connesso ai social network e dedicato al mailing, al microblogging o al chatting, si possono individuare differenze nella lettura di un testo su formato libro e uno su un e-reader?

A questo proposito è molto interessante il nuovo libro di Manfred Spitzer, Demenza digitale, molto radicale nelle sue tesi ma molto interessante per i dati sperimentali che riporta rispetto alla lettura su video.

http://rstampa.pubblica.istruzione.it/utility/imgrs.asp?

numart=2BMB16\&numpag=1\&tipcod=0\&tipimm=1\&defimm=0\&tipnav $=1$

Più in generale, un e-reader è meno efficace dal punto di vista cognitivo, anche quello selfcontained, proprio per la corporeità del libro e la sua tridimensionalità: ad esempio per la lettura fotografica, per il fatto che un libro abbia un retro, una sequenza sinistra-destra e una profondità che aiutano la memorizzazione. Da questo punto di vista, la memoria non è mai 'esterna'. Pensarla in questo modo è come congratularsi con se stessi per il fatto di disporre di una biblioteca di libri non letti. Lessico, empatia, memorizzazione dei contenuti non sono mai disgiunti, penso a quando lavoro per la scrittura saggistica: ho molti libri aperti davanti agli occhi, ho la mia biblioteca davanti a me, entrambi appartengono al mio paesaggio mentale.

Si può dire che in qualche modo l'apologetica del digitale tenda a negare la dimensione corporea? Come se ci fossero menti disincarnate e oggetti privi di significato ergonomico?

Direi di sì: pensiamo erroneamente alla struttura della memoria umana e alle sue differenze con quella di un computer. La memoria del computer ha assegnato degli indirizzi a degli oggetti, è costituita da un grande sistema di meta-dati, in modo che quando cerchi un'informazione vai direttamente a quell'indirizzo, e ovviamente la ritrovi. La memoria umana funziona in modo diverso, è fatta come un sistema di appelli cui tutte le rappresentazioni mentali rispondono, non solo quelle che cerchiamo, sfortunatamente. 
Quando cerchi una cosa in casa, tutte le rappresentazioni di casa tua rispondono in qualche modo: se cerchi le chiavi della macchina, non risponde l'indirizzo del luogo dove le hai lasciate; ma ti risponde tutto, ciascun elemento con una forza diversa che dipende dalla sua forza che a sua volta dipende da vari fattori legati all'aver trattato quell'informazione in profondità. Per questo ti risponde la rappresentazione del portachiavi (usato mille volte) e non trovi le chiavi che erano invece sulla madia della cucina. Quando sei al supermercato, la lista non è semplicemente un promemoria di quello che ti serve, ma è il sistema che ti impedisce di comprare le cose che chiamano costantemente intorno a te e che non ti servono. Ecco, nel mondo in cui tutte le cose chiamano, per fare funzionare un sistema cognitivo come il nostro, bisogna fare un lavoro particolare. Le informazioni sono tanto più attive, quindi in grado di essere chiamate alla coscienza, quanto più sono state trattate in profondità , rielaborate; così riporre le chiavi ogni giorno nello stesso posto funziona, perché quel posto si 'illumina' facilmente quando lo cerchi; ma se cambi posto alle chiavi devi fare qualcosa di specifico per ricordare. Per memorizzare attività poco frequenti bisogna associarle a qualcosa.

\section{Questo discorso vale per l'imparare...}

Imparare significa creare delle rappresentazione mentali organizzate. Queste servono soltanto se te le puoi ricordare, se sono in qualche modo attive nel sistema. Leggere qualcosa senza farci in lavoro di approfondimento è praticamente come non leggere. Noi facciamo empiricamente e a livello elementare un sacco di cose in tal senso: quando sottolineiamo o copiamo a mano, diamo un supplemento di trattamento motorio all'informazione. Quando leggiamo ad alta voce, si tratta di supplemento di trattamento fonetico. Per conoscere un testo, è esperienza elementare, ci lavori, lo rileggi, fai un riassunto. Sono modi di dare ai materiali stoccati in memoria quei 'nastri colorati' che ti permettono di recuperarli facilmente quando ne hai bisogno. L'approfondimento e la rappresentazione viva ti permettono di fare i collegamenti; per questo il libro è straordinario perché ci fai tante cose, lo annoti e lo copi, e lo fai con difficoltà, questo è un punto importante.

Quindi quando lavoriamo per semplificare le nostre didattiche pensando di aiutare $\mathbf{i}$ nostri studenti in realtà stiamo lavorando contro gli effetti che vorremmo ottenere... Lavoriamo contro noi stessi.

Non abbiamo nessun tipo di dato che supporti l'idea, molto diffusa, che bisogna aumentare la semplicità e l'aspetto ludico nell'apprendimento. La scuola è fatica, imparare divertendosi è un concetto insensato, perché comunque imparare non potrà mai essere altrettanto divertente come una partita di calcio. Certo, andare a scuola non deve essere frustrante o punitivo. Il compito della scuola è immenso: si tratta di modificare in pochi anni il cervello delle persone. Per imparare a leggere ci vogliono 2000 ore di lettura; per diventare esperti di qualcosa ci vogliono 5000 ore di attività dedicata. Non è obbligatorio, uno può decidere di non fare entrambe le cose, ma per farlo bisogna sapere che serve lavoro duro, non è una passeggiata. II concetto di " apprendimento facilitato » mi pare un ossimoro.

Altro argomento che poni è la differenza tra lettura superficiale e lettura profonda rispettivamente correlata all'ambiente digitale e cartaceo. 
In termini di scienze cognitive, la lettura non distratta di libri funziona molto bene e dura nel tempo. Marianne Wolf, in Proust e il calamaro, recensisce gli studi che ci dicono chiaramente che la lettura influenza in senso positivo la ricchezza del lessico dei bambini, molto più che la lingua parlata in casa o tra gruppi di amici, assai più povera. È la buona letteratura che migliora le capacità linguistiche e cognitive, e addirittura empatiche ed emotive. Si tratta dunque di proteggere la lettura approfondita, per i vantaggi che questa continua a fornire, dalla concorrenza dei tanti stimoli superficiali e distraenti che vengono dal mondo della connessione digitale; è un compito che la scuola non può demandare.

\section{Nel libro insisti molto sull'importanza dei modelli di design degli ambienti di apprendimento.}

Tra gli equivoci connessi al digitale c'è anche l'idea di ispirarsi alla cultura visiva dei videogames. Esiste una letteratura sui 'benefici del videogames', ma si tratta di tentativi abbastanza disperati di dimostrare che questi migliorino le capacità cognitive. Un videogioco prevede una rapida interazione di tipo senso-motorio, dunque i giochi migliorano gli stimoli di risposta rapida, ma sono attività che possono servire al massimo per l'addestramento di un pilota di droni. E in più è una letteratura 'grigia', legata ai produttori di videogiochi. Mark Prenski, a cui si deve la fortunata definizione di digital natives, è un caso eclatante: che il suo saggio non sia stato preso per quello che era, cioè un depliant pubblicitario burla, ma per un testo scientifico è davvero il segno che il mondo va alla rovescia! II suo è l'argomento di uno sviluppatore di videogiochi, che dice: - Visto che i ragazzi usano molto i videogiochi a casa mettiamoli anche a scuola -. Sarebbe come se un produttore di cioccolato affermasse: - Siccome i ragazzi mangiamo tanti snacks, diffondiamoli anche scuola -. È davvero un pessimo argomento da tutti i punti di vista, dai difetti di logica alla collusione di interessi, ed è semplicemente straordinario che possa aver presa. Una ricerca recente di Milano Bicocca sugli adolescenti in Lombardia ci dice che l'uso del digitale è nei fatti "spippolamento", di sicuro non navigazione nella conoscenza del grande universo. Si riduce a socialnetworking e a scambiarsi il link al video del gatto che tira lo sciacquone; è difficile fare in modo che un uso ludico possa servire a qualcosa di profondamento più impegnativo e diverso.

E poi c'è un altro equivoco didattico estremamente diffuso, che piace molto ai guru e ai ministri, che è quello dell'imparare a imparare. Si tratta di una idea perversa, non si può imparare a imparare, in modo generico. O meglio, se si impara qualcosa (taglio e cucito, programmare in $\mathrm{C}$, trigonometria, storia dell'Armenia, violino o latino) si imparano le strategie che permettono di imparare, ma imparare è sempre e comunque imparare qualcosa. Se tu mi dici: vai in rete e informati sull'osteoporosi o sulla tettonica a placche, in un'ora compongo una bibliografia ragionevole anche se non so nulla di questi temi. Ma noi addetti ai lavori siamo aristocratici della conoscenza perché è una vita che abbiamo imparato a capire quali sono i buoni indici reputazionali: per esempio partiamo da Wikipedia, che ha comunque un buon livello di correttezza, vediamo chi ha scritto sul tema, andiamo sui loro siti e andiamo a vedere le fonti primarie. Per via della nostra professione siamo iperspecializzati e non c'è da sorprendersi che riusciamo poi a trovare informazioni di qualità in rete. Lo possiamo fare perché sappiamo già fare delle cose, che derivano da una formazione precedente: così sappiamo distinguere le fonti e la loro 
pregnanza, gli articoli ben scritti da quelli poco convincenti. Sappiamo cos'è imparare perché abbiamo imparato delle cose a fondo.

In questo senso è importante la distinzione che poni tra informazione e conoscenza.

Sì, come contesto l'idea che l'insegnamento sia solo "trasferimento di conoscenza" e trovo assurdo il concetto di "accesso alla conoscenza". L'accesso deve essere garantito all'informazione, mentre la conoscenza va costruita. Allo stesso modo la creatività non si può insegnare: la creatività è la conseguenza di aver imparato molte cose. I geni sono tutti individui iperspecializzati che hanno sedimentato una quantità enorme di rappresentazioni mentali nel loro settore: 'lì dentro' succede qualcosa. (Controprova : non esistono geni interdisciplinari. Einstein e Nietzsche erano dei geni ed erano dei musicisti, ma erano musicisti modesti.) Ecco quindi l'equivoco: facciamo del male ai nostri studenti se li priviamo della possibilità di specializzarsi in qualcosa, perché li priviamo della possibilità di imparare veramente. Ed è per questo che il digitale lavora esattamente in senso opposto: cerchi tutto, entri dappertutto, giri ed esci come sei entrato, ma sei come un turista che gira in dieci giorni l’Europa. Sei andato dappertutto ma non sei stato da nessuna parte.

In questo senso sei a favore del digitale e contro l' "ideologia digitale".

L'ideologia digitale sostiene la trasmigrazione incondizionata del cartaceo, o di qualsiasi attività non digitale sul digitale, ritenendola preferibile a priori e senza discussione. Attenzione, non è teoria del complotto, la chiamo ideologia in quanto è pronta all'uso, chiunque la può sottoscrivere e fare propria. Sostiene che qualsiasi supporto deve trasmigrare al digitale: la sua unica logica è di sostituzione, o sostituiamo tutto o niente, e quando viene adottata dai policy maker, oltre alla retorica, si accompagna alla logica "del coltello svizzero", lo strumento che in teoria ti permette di fare tutto. In economie di scala e di larga diffusione come in quelle scolastiche si ragiona così: Computer, tablet o e-reader? Prendiamo quello che ci fa fare più cose, così risparmiamo -. Invece bisognerebbe introdurre quello che serve dove serve. Se il libro a scuola funziona meglio in formato cartaceo, lasciamolo in formato cartaceo. Basterebbe una seria sperimentazione, con gruppi di controllo, per rendersi conto di cosa funziona meglio. La logica del coltello svizzero, al contrario, si mangia le logiche più deboli o dedicate, ad esempio preferendo l'acquisto massivo di tablet, piuttosto che di libri o strumenti per le immagini e per la scrittura, digitali e no, o la cura dei laboratori specifici per le diverse materie.

Senza contare che a scuola, nelle pratiche reali e nei capitolati di spesa, la segreteria (registri, assenze etc) passa davanti alla didattica.

Il digitale è un fantastico strumento di controllo, per cui è naturale che l'amministrazione centrale se ne voglia servire: nei fatti viene sempre prima l'informatica burocratica, la parte tecnologica è sempre stata molto legata all'amministrazione.

Aggiungerei che in Italia non esiste una chiara politica ministeriale, o meglio ogni ministero ha perseguito in modo grossolano politiche differenti (LIM, classi 2.0 etc), cambiando programma prima che il precedente fosse in qualche modo compiuto, il tutto a fronte delle carenze gravissime delle infrastrutture necessarie e dei tagli al finanziamento. 
Quando senti parlare gli esperti si avverte una forte stanchezza di fronte all'incessante trasformazione tecnologica. Ma inseguire la tecnologia non è possibile, ci vorrebbero miliardi euro di investimento ogni anno e il settore pubblico non può stare dietro ai colossi del settore. Siamo sommersi di retorica senza supporto empirico. In termini scientifici non sappiamo quasi nulla di come funzionano queste cose. Come possiamo pensarle di iniettarle massicciamente nel sistema didattico? Bisogna sperimentare veramente, ma intanto serve un'applicazione rigorosa del principio di precauzione, che non soltanto ci impone di non applicare quello che è comprovatamente nocivo, ma di non applicare nemmeno quello che non è comprovatamente efficace.

Mi interessa particolarmente la didattica della storia: credo ad esempio che il digitale amplifichi la distanza, senza renderla percepibile, tra il lavoro della ricerca storica e la narrazione della storia.

Non sono uno storico di professione ma posso dire che se rendiamo tutto super-disponibile, la fatica del lavoro storico, della ricostruzione storiografica a partire dalle tracce, sarà sempre più difficile: avremo buoni spettatori e pochi ricercatori. La moderna ricerca scientifica e quella storica nascono insieme accomunate da un rispetto per il dato, che una certa mistica del digitale e della serendipità della rete cancellano in nome di un felice ricerca casuale. Così come scompaiono $i$ mediatori e la competenza degli specialisti. Non c'è capacità di approfondimento ad alto livello senza specialismo.

Il tuo libro contiene anche molte proposte interessanti per reali cambiamenti nel mondo scuola, che prevedono usi intelligenti della tecnologia o diversi approcci alla lettura: «microtutorato verticale» di studenti di scuole superiori che via chat/sms seguono studenti più piccoli; un «mese della lettura» in cui si legge, semplicemente insieme; blog di classe per assistere l'insegnamento; non usare Wikipedia ma scrivere voci di Wikipedia; biblioteche scolastiche «ricche e libere, aperte ai genitori», spazi di lavoro personalizzati. Mi sembra di cogliere l'istanza di una forma attiva di costruttivismo e di una Ricerca-azione 2.0.

Sono solo esempi: Wikipedia ha una diffusione capillare, e fondamentale nella nostra società, non è commerciale, funziona molta bene e chiede che si collabori per tenere una buona qualità dell'informazione. Esserne autori permette di capire come si costruisce l'informazione. Credo si debba intervenire in modo attivo nelle didattiche, non fruendo semplicemente di informazione, ma creando dei percorsi nella tecnologia per produrre conoscenza. 